\title{
Anti-apoptotic oncogenes prevent caspase-dependent and independent commitment for cell death
}

Gustavo P. Amarante-Mendes ${ }^{1,4,6,7}$, Deborah M. Finucane ${ }^{1,6}$, Seamus J. Martin ${ }^{1,5}$, Thomas G. Cotter ${ }^{2}$, Guy S. Salvesen ${ }^{3}$ and Douglas R. Green ${ }^{1,7}$

1 Division of Cellular Immunology, La Jolla Institute for Allergy and Immunology, 10355 Science Center Dr., San Diego, California 92121, USA

2 Tumor Biology Laboratory, Biochemistry Department, University College Cork, Ireland

${ }^{3}$ The Burnham Institute, 10901 North Torrey Pines Road, La Jolla, California, USA

${ }^{4}$ Present address: Department of Immunology, Institute of Biomedical Sciences, University of São Paulo, São Paulo, Brazil

5 Present address: Molecular Cell Biology Laboratory, Department of Biology, Maynooth College, Maynooth, County Kildare, Ireland

${ }^{6}$ These authors have contributed equally to this work

7 corresponding authors: Gustavo P. Amarante-Mendes, PhD, and Douglas R. Green, PhD, Division of Cellular Immunology, La Jolla Institute for Allergy and Immunology, 10355 Science Center Drive, San Diego, California 92121, USA tel: (619)-558-3500; fax: (619)-558-3525

Received 29.9.97; accepted 19.11.97

Edited by R.A. Knight

\begin{abstract}
Apoptosis is a morphologically defined type of cell death associated with the activation of certain proteases belonging to the ICE/CED-3 family, known as caspases. Resistance to apoptosis has been implicated as one of the mechanisms that participates in oncogenesis. We found that the broadspectrum peptide inhibitor of the caspases, ZVAD-fmk, interferes in a dose-dependent way with all the morphological and biochemical changes associated with apoptosis induced by anti-CD95 mAb, staurosporine, VP-16 and Act-D. However, with the exception of anti-CD95-triggered apoptosis, the insulted cells lost their clonogenic potential, even when pretreated with a high dose of zVAD-fmk. Under these circumstances, the dying cells displayed no signs of apoptosis, including activation of caspases, externalization of phosphatidylserine, nuclear condensation, or DNA fragmentation. Instead, this cell death was characterized by cytoplasmic and nuclear vacuolization followed by the loss of plasma membrane integrity. Thus, preventing the onset of apoptosis by blocking caspase activity did not rescue cells from dying in response to drugs such as staurosporine, VP-16 and Act-D. In comparison, ectopic expression of antiapoptotic oncogenes such as bcl-2 and bcr-abl not only inhibited apoptosis but also preserved the clonogenic potential of the cells. Therefore, oncogenesis is promoted not by simply interfering with caspase-mediated apoptosis, but by preventing an upstream event which we define as the commitment point for cell death.
\end{abstract}

Keywords: oncogenes; cell death; apoptosis; caspases; Bcr-Abl; Bcl-2

Abbreviations: PARP, poly(ADP ribose) polymerase; VP-16, etoposide; Act-D, actinomycin-D; PS, phosphatidylserine; $\mathrm{DiOC}_{6}$ [3], 3,3'-dihexyloxacarbocyanine iodide; $\mathrm{mCICCP}$, carbamoyle cyanide n-chlorophenylhydrazone; zVAD-fmk, N-benzyloxycarbonyl-Val-Ala-Asp-fluoromethylketone; $\Delta \Psi_{\mathrm{m}}$, mitochondria inner transmembrane potential

\section{Introduction}

The regulation of apoptotic cell death by oncogenes is thought to be a key to the understanding of cellular transformation, since the balance of cell death and cell proliferation determines whether or not a cell accumulates in the body (Green and Martin, 1995).

Apoptotic cell death is tightly regulated at the molecular level by members of a family of cysteine proteases - the caspases - which cleave specific substrates after aspartic acid residues. Several caspases, also known as ICE/CED3 proteases, constitute the central mechanism of apoptosis, which we call the Executioner (Martin and Green, 1995). Activation of caspases occurs in all forms of apoptosis described so far and is a relatively early event. It precedes or appears concomitantly with all the morphological and biochemical changes specifically associated with apoptosis, such as phosphatidylserine externalization, cell shrinkage, membrane blebbing, nuclear condensation, DNA fragmentation, formation of apoptotic bodies and the proteolytic cleavage of specific substrates like PARP, fodrin, lamins, U1snRNP, D4-GDI, and others (reviewed in Chinnaiyan and Dixit, 1996; Henkart, 1996; Martin and Green, 1995).

Ten members of the human caspase family have been described so far. These molecules are synthesized as proenzymes and await an activating event in the cytosol. Activation results from an internal cleavage that produces a large and small subunit both necessary for the enzymatic activity. Some of the caspases are capable of autoactivation whereas others are only activated by other members of this family, and the existence of an amplification loop or cascade has been postulated (Chinnaiyan and Dixit, 1996; Fraser and Evan, 1996; Henkart, 1996; Martin and Green, 1995). These observations suggest a complex mechanism that governs apoptosis in the higher vertebrates and raise the question of whether all of these molecules share the same, and therefore redundant, role in a universal cell death program or participate in a more specific manner, each individual or sub-group of proteases having a distinct function in the process. 
In the nematode $C$. elegans, mutations in the caspase ced-3 gene result in an increase in living cells due to a loss of developmental cell death (Ellis and Horvitz, 1986; Ellis et al, 1995). Similarly, transgenic expression of the caspase inhibitor p35 from baculovirus in the eye of Drosophila prevents developmental cell death caused by ectopic expression of dE2F and dDP (Nordstrom et al, 1996); or the reaper (White et al, 1996) or hid (Grether et al, 1995) genes. Mice with a homozygous disruption of caspase 3 show a massive accumulation of developing neurons owing to an absence of programmed cell death in the brain (Kuida et al, 1996). Finally, inhibition of caspases by zVAD-DCB administration can limit the extent of neuronal cell death resulting from ischemia and reperfusion (Loddick et al, 1996) and inhibition of caspases with zVAD-fmk prevents cell death after CD95 ligation (Longthorne and Williams, 1997; Xiang et al, 1996). All of these studies argue that caspase activation can represent a cell's decision to live or die.

In apparent opposition to this view, Korsmeyer and colleagues (Xiang et al, 1996) showed that overexpression of Bax induced cell death even in the presence of the caspase inhibitor zVAD-fmk. In addition, Evan and colleagues (McCarthy et al, 1997) showed that peptide inhibitors of caspases prevent some but not all apoptotic morphology and, as a consequence, Rat-1 fibroblasts cannot be rescued from death induced by overexpression of either Bak or Myc or treatment with VP-16. Significantly, this latter study argues that the onset of blebbing coincides with the loss of clonogenic potential suggesting a link between the formation of membrane blebs and the commitment point for cell death.

Based on all these observations and in order to better understand the role of apoptosis in the multi-step tumorigenesis, we decided to compare the ability of zVAD-fmk and the anti-apoptotic oncogenes $b c /-2$ and $b c r-a b /$ to preserve the clonogenic potential of hematopoietic cells treated with different apoptosis-inducing agents.

\section{Results}

\section{zVAD-fmk-inhibitable apoptotic events}

We began our study by defining the chronology of some of the apoptotic events that occur in Jurkat cells after either CD95 ligation or staurosporine treatment. CD95 was recently shown to be directly coupled to a member of the caspase family (caspase 8) which, as a result, acts at a very early stage of the apoptotic signaling. Staurosporine, in itself, is not considered to directly activate any of the caspases; nevertheless it is a very potent and universal inducer of apoptosis. Using these two pathways to launch the apoptotic program in Jurkat cells, we observed a similar sequence of events. Changes in $\Delta \Psi_{\mathrm{m}}$ occurred very early and were followed by activation of caspase 3, PS externalization, nuclear condensation and finally DNA fragmentation (data not shown). zVAD-fmk blocked, in a dose-dependent manner, all the changes associated with apoptosis (Figure 1). This effect was remarkable in CD95-mediated apoptosis but also very significant in staurosporine-treated cells.
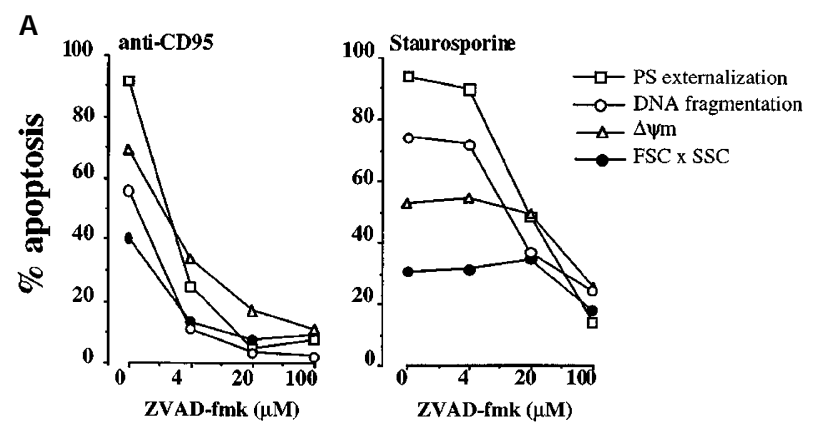

B

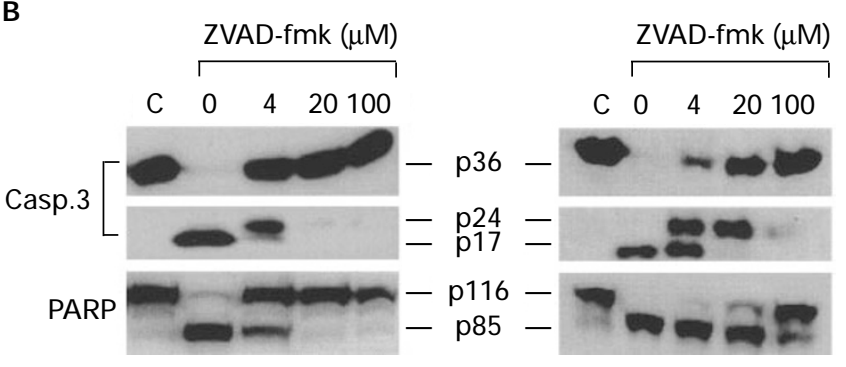

Figure 1 zVAD-fmk blocks anti-CD95- and staurosporine-induced (A) $\Delta \psi_{\mathrm{m}}$, PS externalization, DNA fragmentation, changes in the light scattering properties, (B) activation of caspase 3 and cleavage of PARP. Jurkat cells $\left(1 \times 10^{6}\right)$ were pre-treated for $1: 30 \mathrm{~h}$ with different concentration of $\mathrm{ZVAD}-\mathrm{fmk}$ and subsequently incubated in the presence of anti-CD95 mAbs $(0.75 \mu \mathrm{g} / \mathrm{ml}-$ $9 \mathrm{~h}$ ) or staurosporine $(1 \mu \mathrm{M}-4 \mathrm{~h})$. \% of cell death was estimated according to the techniques described in Materials and Methods

\section{zVAD-fmk-does not prevent drug-induced cell death, only changes its morphology}

Jurkat cells treated with either VP-16, Act-D, staurosporine or anti-CD95 mAb showed signs of apoptosis very rapidly and died in a matter of hours (Figure $2 \mathrm{~A}$ and data not shown). Pretreatment with $100 \mu \mathrm{M}$ zVAD-fmk inhibited the apoptotic process as measured by PS externalization, DNA fragmentation, nuclear condensation and formation of apoptotic bodies (Figures 1 and 2A and data not shown). We then determined the ability of zVAD-fmk to maintain clonogenic potential during treatment with these apoptosis-inducing agents (Figure 2B). These experiments were performed by treating cells with the apoptosis-inducing agents in the presence or absence of zVAD-fmk for periods of time previously established for each agent (4-5 h for staurosporine, VP-16 and Act-D, and 12$16 \mathrm{~h}$ for anti-CD95 mAb treatment). At this point, the level of apoptosis was examined as shown in Figure $2 \mathrm{~A}$. Then, cells were extensively washed and plated at limiting dilution in the presence or absence of fresh zVAD-fmk. Untreated controls contained at least 1 out of 10 cells with the ability to produce clones (all wells showed colonies when cells were plated at 10 cells/well). Anti-CD95 mAb did not kill $100 \%$ of Jurkat cells as observed in the clonogenic assay $(f=0.001)$. In this circumstance, zVAD-fmk allowed cell survival, such that the frequency of cells with clonogenic potential increased by at least two orders of magnitude $(f \geqslant 0.1)$. In contrast, VP-16-, Act-D-, or staurosporine-treated Jurkat cells showed no signs of clonogenicity even in the presence of zVAD-fmk; the frequency of cells able to generate colonies under these 


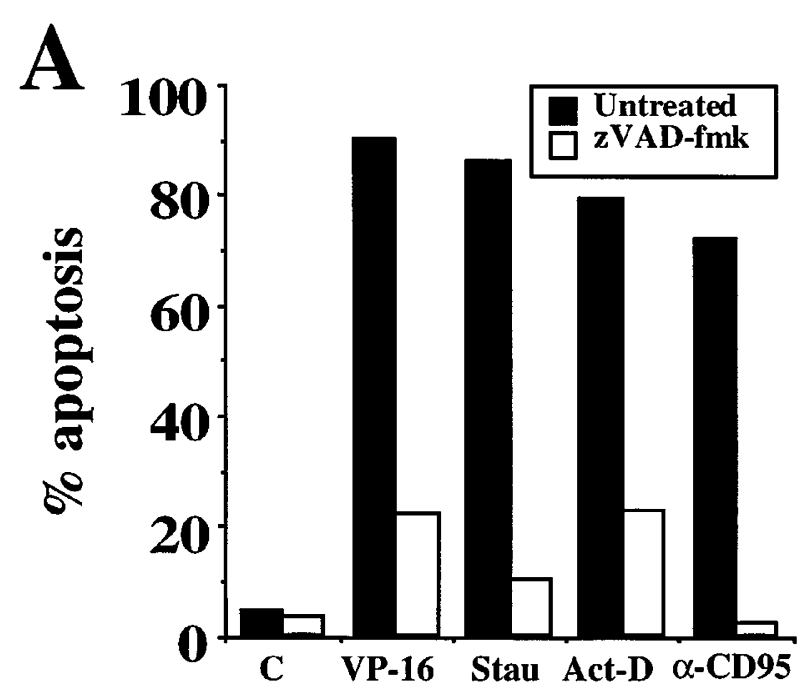

B

\begin{tabular}{|l|cc|}
\cline { 2 - 3 } \multicolumn{1}{c|}{} & None & zVAD-fmk \\
\hline Untreated & $\geq \mathbf{0 . 1}$ & $\geq \mathbf{0 . 1}$ \\
VP16 & $<\mathbf{0 . 0 0 0 2}$ & $<\mathbf{0 . 0 0 0 2}$ \\
Staurosporine & $<\mathbf{0 . 0 0 0 2}$ & $<\mathbf{0 . 0 0 0 2}$ \\
Actinomycin D & $<\mathbf{0 . 0 0 0 2}$ & $<\mathbf{0 . 0 0 0 2}$ \\
anti-CD95 mAbs & $<\mathbf{0 . 0 0 1}$ & $\geq \mathbf{0 . 1}$ \\
\hline
\end{tabular}

\begin{tabular}{|c|c|c|}
\hline & None & zVAD-fmk \\
\hline Untreated & $\geq 0.1$ & $\geq 0.1$ \\
\hline $0.2 \mu \mathrm{M}$ & $\leq 0.01$ & $\leq \mathbf{0 . 0 1}$ \\
\hline $1.0 \mu \mathrm{M}$ & $\leq 0.001$ & $\leq \mathbf{0 . 0 0 1}$ \\
\hline $30 \mathrm{sec}$ & $<0.01$ & $\leq \mathbf{0 . 0 1}$ \\
\hline $1 \mathrm{~min}$ & $\leq \mathbf{0 . 0 0 1}$ & $\leq 0.001$ \\
\hline $2 \mathrm{~min}$ & $<0.001$ & $<0.001$ \\
\hline
\end{tabular}

Figure 2 zVAD-fmk blocks apoptosis but does not prevent cell death. Jurkat cells $\left(1.5 \times 10^{6}\right)$ were treated with either VP-16 $(100 \mu \mathrm{M})$, staurosporine $(1 \mu \mathrm{M})$, Act-D $(1 \mu \mathrm{M})$, or anti-CD95 mAbs $(0.75 \mu \mathrm{g} / \mathrm{ml})$. (A) \% of apoptosis determined by annexin V-binding assay using samples obtained $4-5 \mathrm{~h}$ (for staurosporinetreated cells) or 14-16 h (for VP-16-, Act-D- and anti-CD95-treated cells) after induction of apoptosis. (B) Frequency of cells capable of proliferating after incubation with high doses of apoptosis-inducing agents as determined by the clonogenic assay described in Materials and Methods. (C) Frequency of cells capable of proliferating after incubation with different concentrations of staurosporine or exposed to UV light for different periods of time, estimated as above

circumstances was below detectable levels in our system (less than 1 in 5000 cells). To exclude the possibility that the level of damage induced by these drugs was too great to allow any significant effect of a caspase inhibitor, Jurkat cells were treated with a range of concentrations of staurosporine or submitted to different doses of UV-irradiation, in the presence or absence of zVAD-fmk, and tested for their clonogenic potential. Staurosporine- or UV-treated cells lost, in a dosedependent way, their ability to form colonies regardless of the presence of zVAD-fmk in the culture (Figure 2C). This suggests that even in a limiting situation, the activation of caspases by either staurosporine or UV-irradiation follows the event that commits the cells to die.

Further, we compared the morphological features of cells treated with anti-CD95 mAb or staurosporine, in the presence or absence of zVAD-fmk. By scoring cytospin preparations for viable, necrotic and apoptotic phenotypes, we observed that, for both CD95- (Figure 3A/B) and staurosporine-mediated cell death (Figure 4A/B), Jurkat cells began to show signs of apoptosis at $4-9 \mathrm{~h}$. This morphology was replaced by a secondary necrotic phenotype at $12-18 \mathrm{~h}$, which persisted in latter time points (Figures $3 A / B$ and $4 A / B$ ). zVAD-fmk pre-treatment completely inhibited the appearance of both apoptotic and necrotic cells after incubation with anti-CD95 mAb (Figure $3 A / B)$ and preserved cell viability as observed in the clonogenic assay described above (Figure 2B). In contrast, zVAD-fmk only delayed the loss of viability induced by the transient staurosporine treatment (Figure 4A/B). In this situation, Jurkat cells almost exclusively exhibited a necrotic-like phenotype with no signs of apoptosis. The efficacy of the zVAD-fmk was confirmed by biochemical analysis of the activation of caspases. Caspase 3 was activated following treatment with anti-CD95 or staurosporine as revealed by immuno blot analysis (Figures $3 \mathrm{C}$ and $4 \mathrm{C})$. The disappearance of the p36 pro-form occurred early in staurosporine-induced apoptosis compared to anti-CD95induced death and correlated with the appearance of the p17 subunit of the activated caspase 3 as described (Martin et al, 1996). With time, p17 also became undetectable in both cases, probably as a consequence of degradation due to secondary necrosis. Cleavage of PARP confirmed the activation of caspases. zVAD-fmk blocked the activation of caspase 3 by staurosporine for at least $60 \mathrm{~h}$, at which time $60-80 \%$ of the cells were necrotic by morphology (Figure $4 \mathrm{~A} / \mathrm{B}$ ) and confirmed by PI uptake (not shown). These observations strongly argue against the participation of caspases in the observed cell demise under these circumstances.

\section{Anti-apoptotic oncogenes inhibit apoptosis and prevent cell death}

We then tested the ability of anti-apoptotic oncogenes to preserve clonogenicity after apoptotic stimulation. Jurkat cells overexpressing $\mathrm{Bcl}-2$ showed strong resistance to apoptosis when compared to vector controls in a short-term assay (Figure 5A). Staurosporine-treated and untreated Jurkat. Bcl2 exhibited similar frequency of cells with clonogenic potential $(f \geqslant 0.1)$, which indicates that Bcl-2 acts upstream of the commitment point for cell death initiated by staurosporine (Figure 5B). In contrast, Bcl-2 overexpression was not sufficient to prevent VP-16- and ACt-D-induced loss of clonogenicity $(f \leqslant 0.0002)$. Despite the resistance to apoptosis observed at early time points, and in contrast to the zVADfmk treatment, overexpression of $\mathrm{Bcl}-2$ only delayed the appearance of the apoptotic phenotype after treatment with 


\section{A}

\section{Anti-CD95}

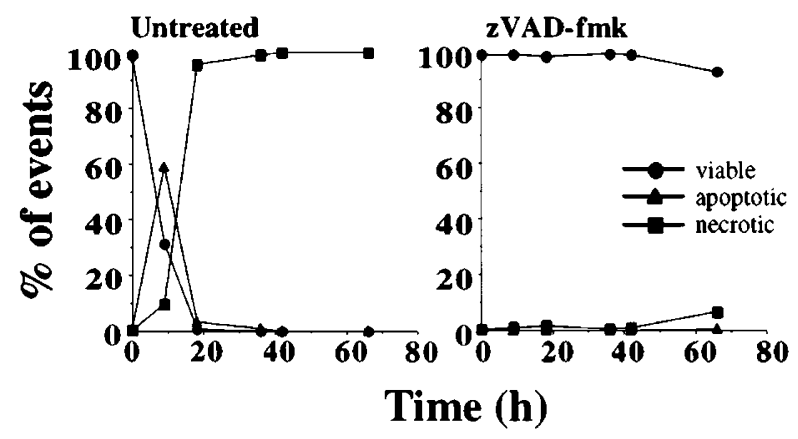

B
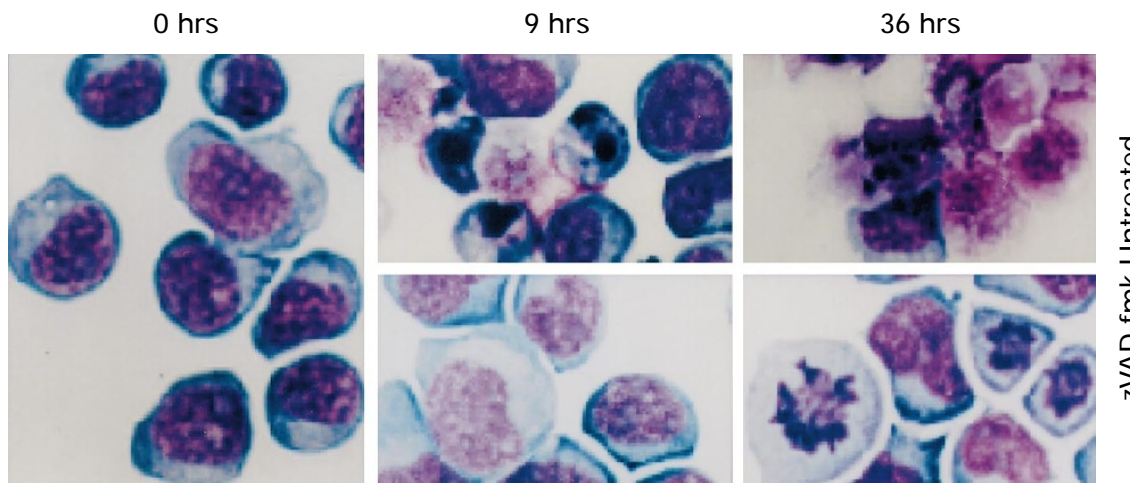

C

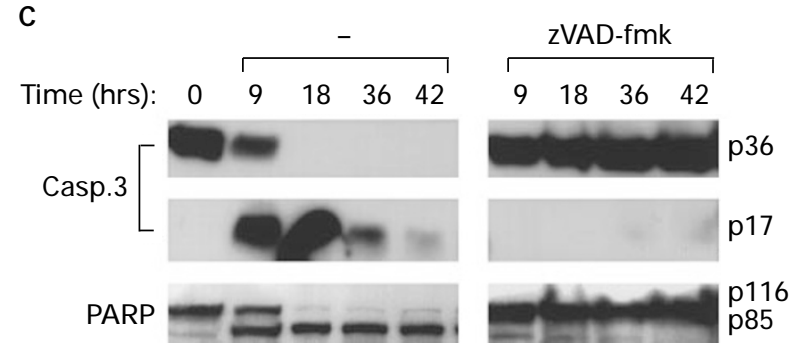

Figure 3 zVAD-fmk blocks CD95-mediated apoptosis and preserve cell viability. (A) \% of viability, necrosis and apoptosis were verified in citospun preparations of Jurkat cells treated with anti-CD95 mAbs $(0.75 \mu \mathrm{g} / \mathrm{ml})$ in the presence or absence of zVAD-fmk. (B) zVAD-fmk preserve cell morphology in anti-CD95-treated cells. Photographs show representative fields of samples obtained in at least three independent experiments. (C) Activation of caspase 3 and cleavage of PARP, as determined by Western-blot, do not occur in Jurkat cells pre-treated with zVAD-fmk

VP-16 or Act-D. Thus, Bcl-2 appears to prevent the initiation of the event(s) responsible for the commitment to death in certain circumstances but only to delay it in others.

We have previously observed that ectopic expression of another oncogene, $b c r-a b l$, in the promyelocytic cell line HL-60 cells confers a remarkable resistance to apoptosis regardless of the inducing agent (Amarante-Mendes et al, in press). We used this system to investigate the ability of this tyrosine-kinase to preserve the clonogenic potential of cells induced to undergo apoptosis. As with Jurkat cells, we found that pre-treatment with ZVAD-fmk protects HL-60 cells from apoptosis (Figure 6A) but did not rescue them from death (Figure 6B). Remarkably, expression of Bcr-Abl in HL-60 cells not only prevented the onset of apoptosis but also maintained the clonogenic potential, thus completely protecting from death induced by VP-16, staurosporine and anti-CD95 mAb (Figure 6C). Together, these data show that the anti-apoptotic oncogenes do not act merely by preventing the onset of apoptosis but by blocking an upstream event that commits the cell to either caspasedependent or independent cell death.

\section{Discussion}

The growth of a tissue is a function not only of cell proliferation but also of cell death. This is especially relevant to cancer, where the loss of controls on the growth or death of one cell can compromise the integrity of the entire individual. Several 


\section{A}

\section{Staurosporine}

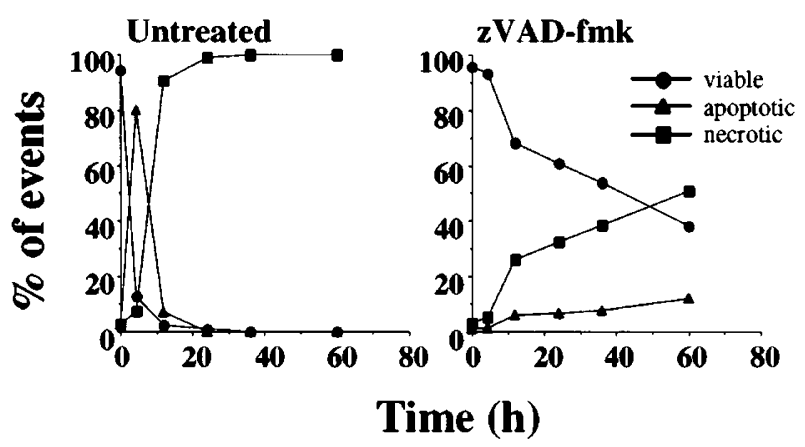

B
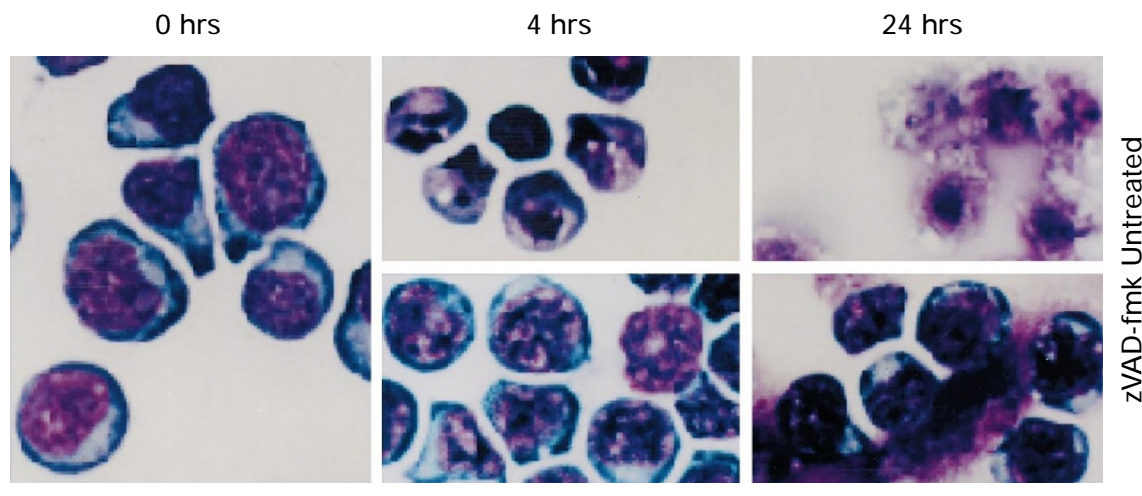

C

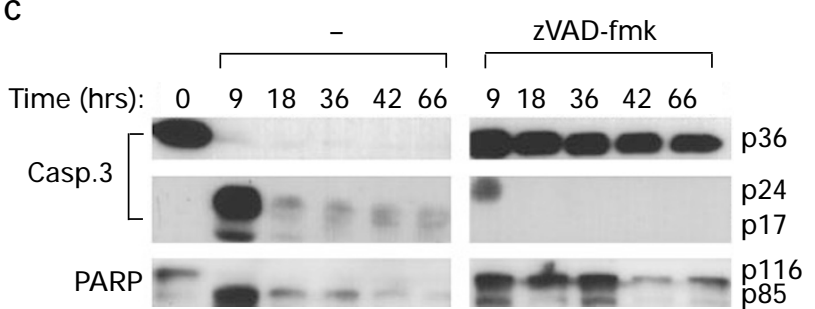

Figure 4 zVAD-fmk blocks staurosporine-induced apoptosis but does not preserve cell viability. (A) \% of viability, necrosis and apoptosis were verified in citospun preparations of Jurkat cells treated with staurosporine $(1 \mu \mathrm{M})$ in the presence or absence of zVAD-fmk. (B) zVAD-fmk delays cell death induced by staurosporine and changes its morphology. Photographs show representative fields of samples obtained in at least three independent experiments. (C) Activation of caspase 3 and cleavage of PARP, as determined by Western-blot, do not occur in Jurkat cells pre-treated with zVAD-fmk even after $60 \mathrm{~h}$ after staurosporine treatment

oncogenes have been shown to inhibit apoptosis, and it is likely that this effect is an important contributor to transformation (Green and Martin, 1995). Thus, an understanding of how an oncogene inhibits apoptosis is of major importance for understanding the phenomenon of cancer.

As outlined in the Introduction, caspases play a central role in orchestrating the biochemical events in apoptotic cell death. It is noteworthy, then, that anti-apoptotic oncogenes such as Bcl-2 (Chinnaiyan et al, 1996; Shimizu et al, 1996) and Bcr-Abl function to inhibit activation of caspases during apoptosis. This may seem, at a first glance, the most likely mechanism to account for the resistance to cell death observed in some forms of cancer. However, this can only be true if the activation of the executioner caspases, such as caspase 3 , precedes the point in the cell death pathway at which the cells are committed to die.

Pharmacological inhibition of caspases by zVAD-fmk in the human hematopoietic cell lines Jurkat and HL-60 prevented the morphological and biochemical changes associated with apoptosis but did not preserve viability after treatment with staurosporine, VP-16, Act-D or UVirradiation. These cells died with an unusual morphology characterized by extensive cellular vacuolization accompanied by a delayed loss of cell membrane integrity. These data are in agreement with previous reports (McCarthy et al, 1997; Xiang et al, 1996) and support the view that cells 
A

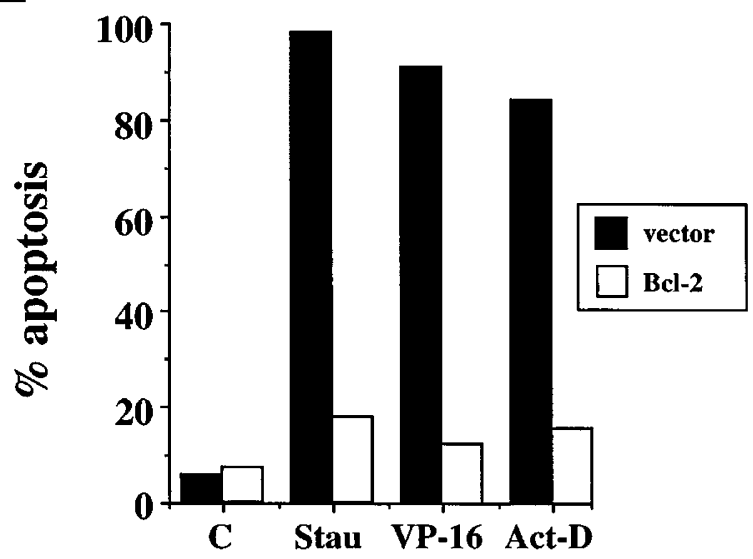

$\mathbf{B}$

\begin{tabular}{|l|cc|}
\cline { 2 - 3 } \multicolumn{1}{c|}{} & vector & Bcl-2 \\
\hline Untreated & $\geq 0.1$ & $\geq 0.1$ \\
VP16 & $<\mathbf{0 . 0 0 0 2}$ & $<\mathbf{0 . 0 0 0 2}$ \\
Actinomycin D & $<\mathbf{0 . 0 0 0 2}$ & $<\mathbf{0 . 0 0 0 2}$ \\
Staurosporine & $<0.0002$ & $\geq 0.1$ \\
\hline
\end{tabular}

Figure 5 Ectopic expression of $\mathrm{Bcl}-2$ protects Jurkat cells from staurosporine- but not from VP-16- or Act-D-induced cell death. Jurkat and Jurkat.Bcl-2 cells $\left(1.5 \times 10^{6}\right)$ were treated with either staurosporine $(1 \mu \mathrm{M})$ VP-16 $(100 \mu \mathrm{M})$ or Act-D $(1 \mu \mathrm{M})$ for particular time periods, washed and plated in 96-well tissue culture plates. (A) \% of apoptosis determined by annexin Vbinding assay using samples obtained $4-5 \mathrm{~h}$ (for staurosporine-treated cells) or 14-16 h (for VP-16- and Act-D-treated cells) after induction of apoptosis. (B) Frequency of cells capable of proliferating after incubation with apoptosisinducing agents determined by the clonogenic assay as described in Materials and Methods

may commit to die before the activation of the caspases. It is formally possible, however, that even the broad-spectrum caspase inhibitors such as zVAD-fmk may not block all caspases or may do so only temporarily, and therefore the apparent caspase-independent cell death is actually due to either the effects of another unknown protease or to a delayed activation of the described caspases. We consider this unlikely however, since none of the caspasedependent biochemical and morphological changes normally associated with apoptosis were observed in the dying cells pre-treated with zVAD-fmk. Further, the failure of this caspase inhibitor to increase clonogenicity even when the insult was limited suggests that none of the biochemical events required for the commitment to cell death are even partially sensitive to inhibition by zVAD-fmk.

The anti-apoptotic oncogenes, Bcl-2 and Bcr-Abl produced a very different effect with respect to these apoptosis-inducing agents. Not only was apoptosis inhibited, but in most cases cell death was blocked in cells expressing these proteins, such that cells maintained clonogenicity. Again, the failure of the caspase inhibitor to block cell death (as opposed to apoptosis) suggests that
A

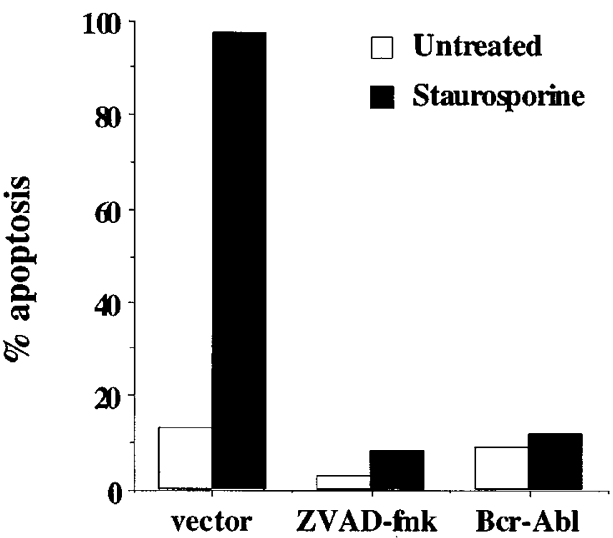

B

\begin{tabular}{|l|ccc|}
\cline { 2 - 4 } \multicolumn{1}{c|}{} & vector & ZVAD-fmk & Bcr-Abl \\
\hline Untreated & $\geq 0.1$ & $\geq 0.1$ & $\geq 0.1$ \\
Staurosporine & $<\mathbf{0 . 0 0 0 2}$ & $<\mathbf{0 . 0 0 0 2}$ & $\geq 0.1$ \\
\hline
\end{tabular}

C

\begin{tabular}{|l|cc|}
\cline { 2 - 3 } \multicolumn{1}{c|}{} & vector & Bcr-Abl \\
\hline Untreated & $\geq 0.1$ & $\geq 0.1$ \\
VP16 & $<0.0002$ & $\geq 0.1$ \\
anti-CD95 Abs & $<0.0002$ & $\geq 0.1$ \\
Staurosporine & $<0.0002$ & $\geq 0.1$ \\
\hline
\end{tabular}

Figure 6 Bcr-Abl inhibits apoptosis and provide clonogenic potential. HL-60 cells pre-treated or not with $100 \mu \mathrm{M}$ zVAD-fmk and HL-60.Bcr-Abl cells were incubated with staurosporine $(1 \mu \mathrm{M})$, VP-16 $(100 \mu \mathrm{M})$ or anti-CD95 mAb $(1 \mu \mathrm{g} /$ $\mathrm{ml}$ ) for different periods of time, washed and plated in 96-well tissue culture plates. (A) \% of apoptosis on staurosporine-treated samples as determined by annexin V-binding assay. (B and $\mathbf{C}$ ) Frequency of cells capable of proliferating after incubation with apoptosis-inducing agents

direct inhibition of caspases may not be a route to cell survival, at least in some circumstances. In addition, it indicates that the activity of anti-apoptotic oncogenes must lie at some point upstream of caspase activation, prior to a point that commits the cell to die regardless of whether caspases are engaged in the pathway. It is also important that in the cases that $\mathrm{Bcl}-2$ was unable to provide clonogenicity to the insulted cells, these died with apoptotic morphology. Therefore, caspase-dependent apoptosis is not a requirement for cell death but it is the preferable (or fastest) way to fulfill the cell's destiny.

How then can we resolve the apparent paradox between these conclusions and the observations in several developmental models suggesting that caspases are not only important for orchestrating the apoptotic phenotype, but also control whether or not cells die (Ellis and Horvitz, 1986; Ellis et al, 1995; Grether et al, 1995; Kuida et al, 1996; Nordstrom et al, 1996; White et al, 1996). One possible explanation comes from the idea that there are at least two distinct routes to committing a cell to die (Figure 7). In some cases, including CD95 and presumably those forms of programmed cell death in which caspases play a 


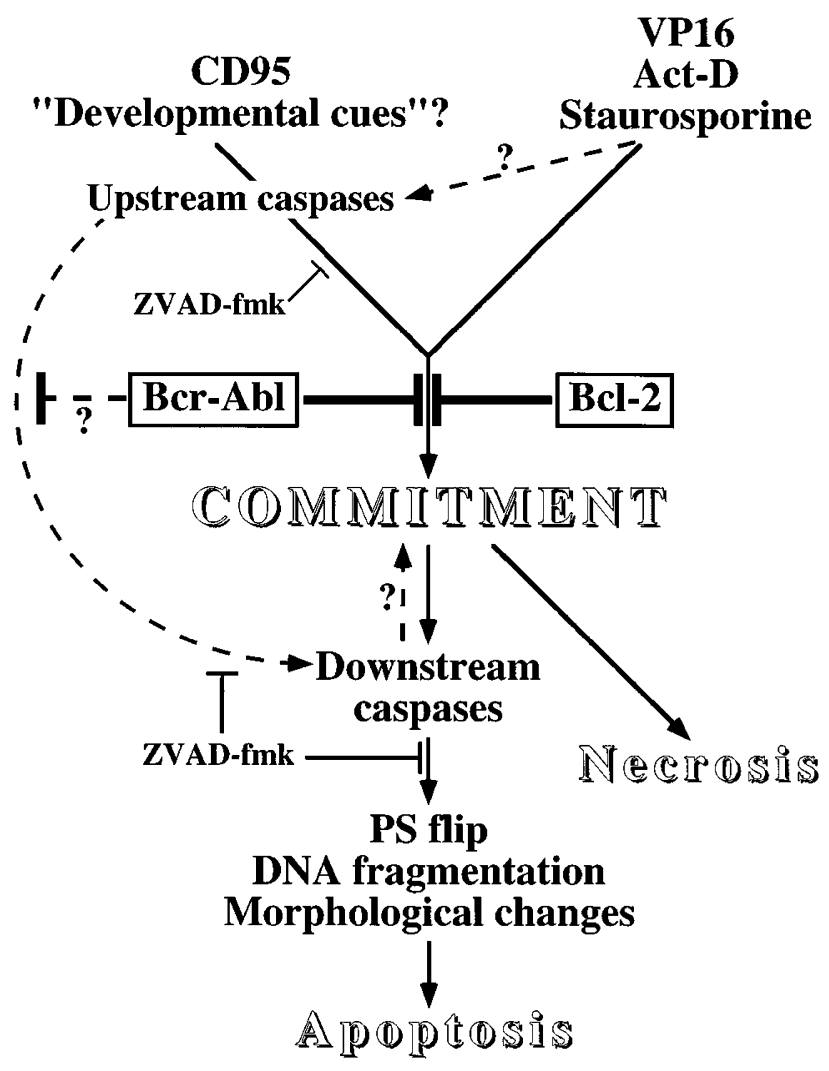

Figure 7 Scheme of pathways involved in cell death. Signals coming from initially distinct pathways for cell death, here represented by the two major groups - 'developmental cues' and 'stress signaling' - converge to the point of no return. When the cells sense the commitment to death, they activate the apoptotic machinery to speed up the death process and package themselves in such a way to avoid any inflammatory reaction. Inhibitors of the executioner caspases completely block the events correlated with apoptosis but are unable to rescue the cells that have already been commited to die. Exception to this rule is provided by apoptotic signals derived from CD95 or developmental cues, in which case inhibitors of the caspases prevent cell demise. These observations suggest the existence of initiating (or upstream) and executioner (or downstream) caspases. Oncogenes such as Bcr-Abl and Bcl-2 block the apoptotic signal before the commitment point thus preventing the activation of the executioner caspases

crucial role (mentioned above), initiating (or upstream) caspases are required to launch the pathway that leads to the Commitment Point. In other cases, such as those described here and by others (McCarthy et al, 1997; Xiang et al, 1996), other cellular stresses act independently of such regulatory caspases to commit the cells to die, following which the activation of the executioner caspases ensures that the death proceeds in an accelerated and ordered fashion via apoptosis (for example, see Figure 7).

To support our view, it has been suggested (Kumar and Lavin, 1996) that molecules that contain a larger prodomain, such as caspase 8 (FLICE/MACH-1/Mch-5), caspase $2(\mathrm{ICH}-1)$ and caspase 1 (ICE) act as upstream signals regulating the initiation of the apoptotic program whereas molecules with a small pro-domain, particularly the ones belonging to the caspase 3 (CPP32/Yama/Apopain) sub-family, would be downstream proteases ('execu- tioners') directly responsible for coordinating apoptotic events. In the scheme shown in Figure 7, initiator caspases would be those that lie upstream of the commitment point following some forms of apoptosis induction, while the executioner caspases would be those that lie downstream of commitment. Anti-apoptotic oncogenes can interfere with both forms of commitment, resulting in cell survival.

How do anti-apoptotic oncogenes function in this regard? It is tempting to speculate that both Bcl-2 and Bcr-Abl function to block death via either pathway through one (and possibly the same) site of action, the 'Commitment Point' in Figure 7. In those cases where anti-apoptotic effects of Bcl2 are not observed (e.g., Bcl-2 fails to block CD95mediated apoptosis in some cells (Strasser et al, 1995)) it is unclear whether this represents a bypass of the commitment point, an inactivation of the anti-apoptotic function, or both, and these are not considered in the figure.

Despite the vast literature dealing with anti-apoptotic oncogenes, in particular those belonging to the $\mathrm{Bcl}-2$ family, little is known about the mechanisms used by these molecules to prevent apoptosis. Some clues were recently provided by the observations that the structure of $\mathrm{Bcl}-\mathrm{x}_{\mathrm{L}}$ resembles the diphtheria toxin, which is able to form channels in cellular membranes (Muchmore et al, 1996), and that Bcl- $\mathrm{x}_{\mathrm{L}}$ (Minn et al, 1997) and Bcl-2 (Schendel et al, 1997) can indeed form ion channels in vitro. These findings are very intriguing in light of another set of observations placing mitochondria and mitochondria-derived factors as coordinating events in apoptosis (Petit et al, 1995; Susin et al, 1996; Yang et al, 1997). In this regard, two molecules, AIF (apoptosis-inducing factor) and cytochrome c, have been linked to the onset of apoptosis. Both were shown to be released from mitochondria during apoptosis, to precede the appearance of the morphological and biochemical changes associated with this form of cell death and to trigger apoptosis in cell-free systems (Susin et al, 1996; Yang et al, 1997). Most interestingly, the survival-promoting proto-oncogene $b c l-2$ was recently shown to inhibit the release of these factors (Kluck et al, 1997; Susin et al, 1996; Yang et al, 1997), supporting the existence of a check-point for apoptosis at the mitochondrial level (see Henkart and Grinstein, 1996). Taken together, these observations suggest that mitochondria are the target of signals coming from initially distinct pathways that lead to apoptosis and, as a consequence of such stimuli, initiate the common pathway coordinated by the executioner caspases.

Whether AIF, cytochrome c, or the mitochrondrial permeability transition (PT) pores account for the commitment to cell death is still a matter of debate. It has been suggested that the loss of $\Delta \Psi_{\mathrm{m}}$, which may be due to the opening of PT pores, is a central and irreversible step in apoptosis (Marchetti et al, 1996; Petit et al, 1995; Vayssiere et al, 1994; Zamzami et al, 1995). We observed that in Jurkat cells, changes in mitochondrial transmembrane potential occur very early, before detection of caspase activity. However, the fact that zZAD-fmk can block $\Delta \Psi_{\mathrm{m}}$ (Figure 1 and Marchetti et al, 1996) suggests that caspases 
do participate in the loss of mitochondrial transmembrane potential in these cells, and challenges the view that $\Delta \psi_{\mathrm{m}}$ is the only commitment point for cell death. In support of this, we found no correlation between $\Delta \psi_{\mathrm{m}}$ and apoptosis in $\mathrm{HL}$ 60 cells (DM Finucane, GP Amarante-Mendes, TG Cotter and DR Green. Collapse of the inner mitochondrial transmembrane potential is not a universal requirement for apoptosis. Unpublished observations).

In these cells, $\Delta \Psi_{\mathrm{m}}$ does not occur or is very delayed in response to apoptosis-inducing agents. In addition, we consistently observed HL-60 apoptotic bodies showing bright mitochondrial staining using the $\mathrm{DiOC}_{6}[3]$ dye. Similarly, we and others have found that the release of cytochrome c from mitochondria during apoptosis occurs independently of the mitochondrial permeability transition (Kluck et al, 1997; Yang et al, 1997; Bossy-Wetzel et al, in press) and it is not unreasonable to speculate that this release may be a consequence of an irreversible change in mitochondria that commits the cell to die.

Since inhibitors of the caspases prevent apoptosis but do not allow cellular proliferation or survival under the conditions we have analyzed, a simple interference in the caspase machinery is not enough to account for the resistance to chemotherapeutic treatment that some oncogenes confer. We showed here that, in fact, Bcl-2 and Bcr-Abl not only suppress the onset of apoptosis but must also act by blocking the event that commits the cells to die. Understanding this 'Commitment Point' will help to unravel the molecular mechanism(s) that activate the executioner caspases and will provide new insights for anti-cancer therapy.

\section{Materials and Methods}

\section{Cell culture and reagents}

All cells were cultured in RPMI-1640 supplemented with $10 \%$ FCS and 2 mM L-glutamine (RPMI-FCS). Jurkat.Bcl-2 (Martin et al, 1995a) and HL-60.Bcr-Abl cells (Amarante-Mendes et al, 1997) were described elsewhere.

VP-16 staurosporine were prepared as $100 \mathrm{mM}$ and $1 \mathrm{mM}$ solutions in dimethyl sulfoxide (DMSO), respectively. Act-D was prepared as $1 \mathrm{mM}$ stock solution in RPMI-1640. Anti-CD95 IgM mAb (Kamiya Biomedical Co., Thousand Oaks, CA) was prepared as $0.1 \mathrm{mg}^{-1}$ stock solution in PBS. zVAD-fmk was obtained from Kamiya and prepared as $20 \mathrm{mM}$ stock solution in DMSO. Annexin-V was obtained from BioWhittaker, Inc., Walkersville, MD, $\mathrm{DiOC}_{6}[3]$ and $\mathrm{mCICCP}$ was purchased from Molecular Probes, Inc., Eugene, OR.

Anti-caspase 3 and anti-PARP polyclonal antibody was generously provided by Dr John Reed (Burnham Institute, La Jolla, CA) and Dr Carlos Casiano (The Scripps Research Institute, La Jolla, CA), respectively.

\section{Western-blot analysis}

Cells were harvested, washed once in cold PBS, lysed directly in SDSsample buffer (2.5\% 2-ME, 2\% SDS, $10 \%$ glycerol, $50 \mathrm{mM}$ Tris-Cl $\mathrm{pH} 6.8$ ) and immediately boiled for $5 \mathrm{~min}$. Samples were resolved on SDS-polyacrylamide gel, transferred to PVDF membranes and immunoblotted with different primary antibodies. Reactions were detected with suitable secondary antibody conjugated to horseradish peroxidase using an enhanced chemiluminescence system (ECL, Amersham).

\section{Determination of apoptosis}

Apoptosis was assessed by different criteria. PS externalization was determined by the Annexin-V-binding assay as previously described (Martin et al, 1995b).

Changes in light scattering properties due to reduction in cell size (FSC analysis) and increase in granularity (SSC analysis) as consequence of apoptosis (McGahon et al, 1995) were determined in a FACScan flow cytometer (Becton-Dickinson, Mountain View, CA) in the same samples acquired for the Annexin-V-binding assay.

Collapse of the inner mitochondrial transmembrane potential $\left(\Delta \psi_{\mathrm{m}}\right)$ was measured using the $\mathrm{DiOC}_{6}[3]$ dye. Briefly, cells were washed once and incubated in $200 \mu$ of PBS containing $40 \mathrm{nM}$ of $\mathrm{DiOC}_{6}[3]$ at $37^{\circ} \mathrm{C}$ for $20-30$ min before analyzed in a flow cytometer. Controls included cells treated with the mitochondrial uncoupler $\operatorname{mCICCP}(50 \mu \mathrm{M})$.

DNA fragmentation was quantified as the percentage of nuclei (Nicoletti et al, 1991). In brief, $2 \times 10^{5}$ cells were placed in a well of a 96-well round-bottomed tissue-culture plate, spun down and resuspended in $200 \mu \mathrm{l}$ of a hypotonic fluorochrome solution $(50 \mu \mathrm{M}$ propidium iodide in $0.1 \%$ sodium citrate $-0.1 \%$ Triton $\mathrm{X}-100)$. The suspensions were then incubated on ice for 30-60 min and analyzed in a FACScan flow cytometer.

Morphological evaluation of the cell death was accomplished in cytospun preparations (McGahon et al, 1995). Apoptosis was evaluated by nuclear condensation, cell shrinkage and membrane blebbing. Necrosis was scored by loss of nuclear staining, absence of nuclear condensation, loss of plasma membrane integrity and presence of cell 'ghost'.

\section{Clonogenic assay}

Cells were treated or not for 90 min with zVAD-fmk and incubated with apoptosis-inducing agents for different periods of time. The duration of the incubation with the inducing agents was established beforehand as the time when we observed high level of apoptosis occurring in zVADuntreated but not in ZVAD-treated cells. Then, cells were washed three times in large volumes of HBSS, resuspended in fresh medium, and plated in 96-well round-bottomed tissue culture plates. In every case, samples were plated at 10, 100, 1000 and 5000 cells/well in 24 replicates. The frequency of cells with clonogenic potential were estimated after 14 days by counting positive wells. In most of the cases, the results were very distinctive; either we observed formation of colonies in every well even when samples were plated at 10 cells/ well $(f \geqslant 0.1)$ or none of the well showed any sign of growth even when samples were plated at 5000 cells/well $(f \leqslant 0.0002)$.

\section{Acknowledgements}

We would like to thank Drs John Reed (The Burnham Institute, La Jolla, CA) and Carlos Casiano (The Scripps Research Institute, La Jolla, CA) for the gifts of anti-caspase 3 and anti-PARP antibodies, respectively, and $\mathrm{Dr}$ Thomas Brunner for helpful discussion. GPA-M was a Brazilian Research Council (CNPq) Fellow. DMF is supported in part by The Children Leukemia Research Project fellowship. SJM was a Wellcome Trust International Traveling Fellow (041080). This research was supported by grants from the American Cancer Society (CB-82) and the National Institutes of Health (CA69381 and Al40646) to DRG. This is the publication \#188 from the La Jolla Institute for Allergy and Immunology. 


\section{References}

Amarante-Mendes GP, Jascur T, Nishioka WK, Mustelin T and Green DR (1997) BcrAbl-mediated resistance to apoptosis is independent of PI 3-kinase activity. Cell Death Differ. 4: 548-554

Amarante-Mendes GP, McGahon AJ, Nishioka WK, Afar DEH, Witte ON and Green DR. Bcl-2-independent Bcr-Abl-mediated resistance to apoptosis: Protection is correlated with up-regulation of $\mathrm{Bcl}-\mathrm{x}_{\mathrm{L}}$. Oncogene in press

Bossy-Wetzel E, Newmeyer DD and Green DR. Mitochondrial cytochrome c release in apoptosis occurs upstream of DEVD-specific caspase activation and independently of mitochondrial transmembrane potential. EMBO J. in press

Chinnaiyan A and Dixit V (1996) The cell-death machine. Curr. Biol. 6: 555-562

Chinnaiyan AM, Orth K, O'Rourke K, Duan H, Poirier GG and Dixit VM (1996) Molecular ordering of the cell death pathway: $\mathrm{Bcl}-2$ and $\mathrm{Bcl}-\mathrm{xL}$ function upstream of the CED-3-like apoptosis proteases. J. Biol. Chem. 271: 4573-4576

Ellis HM and Horvitz HR (1986) Genetic control of programmed cell death in the nematode C. elegans. Cell 44: 817-829

Ellis RE, Yuan JY and Horvitz HR (1995) Mechanisms and functions of cell death. Annu. Rev. Cell Biol. 7: 663-698

Fraser A and Evan G (1996) A licence to kill. Cell 85: 781-784

Green DR and Martin SJ (1995) The killer and the executioner: how apoptosis controls malignancy. Curr. Opin. Immunol. 7: 694-703

Grether ME, Abrams JM, Agapite J, White Kand Steller H(1995) The head involution defective gene of Drosophila melanogaster functions in programmed cell death. Genes Dev. 9: $1694-1708$

Henkart PA (1996) ICE family proteases: mediators of all apoptotic cell death? Immunity 4: 195-201

Henkart PA and Grinstein S (1996) Apoptosis: mitochondria resurrected? J. Exp. Med. 183: 1293-1295

Kluck RM, Bossy-Wetzel E, Green DR and Newmeyer DD (1997) The release of cytochrome $\mathrm{c}$ from mitochondria: a primary site for $\mathrm{Bcl}-2$ regulation of apoptosis. Science 275: 1132-1136

Kuida K, Zheng TS, Na S, Kuan C-Y, Yang D, Karasuyama H, Rakic P and Flavell RA (1996) Decreased apoptosis in the brain and premature lethality in CPP32deficient mice. Nature 384: 368-372

Kumar S and Lavin MF (1996) The ICE family of cysteine proteases as effector of cell death. Cell Death Differ. 3: 255-267

Loddick CA, MacKenzie A and Rothwell NJ (1996) An ICE inhibitor, z-VAD-DCB attenuates ischaemic brain damage in the rat. Neuroreport 7: 1465-1468

Longthorne VL and Williams GT (1997) Caspase activity is required for commitment to Fas-mediated apoptosis. EMBO J. 16: 3805-3812

Marchetti P, Castedo M, Susin SA, Zamzami N, Hirsch T, Macho A, Haeffner A, Hirsch F, Geuskens M and Kroemer G (1996) Mitochondrial permeability transition is a central coordinating event of apoptosis. J. Exp. Med. 184: 1155-1160

Martin SJ, Amarante-Mendes GP, Shi L, Chuang TH, Casiano CA, O'Brien GA, Fitzgerald P, Tan EM, Bokoch GM, Greenberg AH and Green DR (1996) The cytotoxic cell protease granzyme $B$ initiates apoptosis in a cell-free system by proteolytic processing and activation of the ICE/CED-3 family protease, CPP32, via a novel two-step mechanism. EMBO J. 15: 2407-2416

Martin SJ and Green DR (1995) Protease activation during apoptosis: death by a thousand cuts? Cell 82: 349-352

Martin SJ, Reutelingsperger CPM, McGahon AJ, Rader J, van Schie RCAA, LaFace DM and Green DR (1995a) Early redistribution of plasma membrane phosphatidylserine is a general feature of apoptosis regardless of the initiating stimulus: inhibition by overexpression of Bcl-2 and Abl. J. Exp. Med. 182: 1-12
Martin SJ, Takayama S, McGahon AJ, Miyashita T, Corbeil J, Kolesnick RN, Reed JC and Green DR (1995b) Inhibition of ceramide-induced apoptosis by Bcl-2. Cell Death Differ. 2: 253-257

McCarthy NJ, Whyte MKB, Gilbert CS and Evan GI (1997) Inhibition of Ced-3/ICErelated proteases does not prevent cell death induced by oncogenes, DNA damage, or the Bcl-2 homologue Bak. J. Cell Biol. 136: 215-227

McGahon AJ, Martin SJ, Mahboubi A, Bissonette RP, Shi Y, Mogil R, Nishioka W and Green DR (1995) The end of the (cell) line: Methods for the study of apoptosis in vitro. In Cell Death, LM Schwartz and BA Osborne, (eds) (San Diego: Academic Press, Inc.), pp. 153-186

Minn AJ, Velez P, Schendel SL, Liang H, Muchmore SW, Fesik SW, Fill M and Thompson CB (1997) Bcl-x(L) forms an ion channel in synthetic lipid membranes. Nature 385: 353-357

Muchmore SW, Sattler M, Liang H, Meadows RP, Harlan JE, Yoon HS, Nettesheim D, Chang BS, Thompson CB, Wong SL, Ng S-C and Fesik SW (1996) X-ray and NMR structure of human $\mathrm{Bcl}-\mathrm{xL}$, an inhibitor of programmed cell death. Nature 381: $335-341$

Nicoletti I, Migliorati G, Pagliacci MC, Grignani F and Riccardi C (1991) A rapid and simple method for measuring thymocyte apoptosis by propidium iodide staining and flow cytometry. J. Immun. Meth. 139: 271-279

Nordstrom W, Chen P, Steller H and Abrams JM (1996) Activation of the reaper gene during ectopic cell killing in Drosophila. Dev. Biol. 180: 213-226

Petit PX, Lecoeur H, Zorn E, Dauguet C, Mignotte B and Gougeon ML (1995) Alterations in mitochondrial structure and function are early events of dexamethasone-induced thymocyte apoptosis. J. Cell Biol. 130: 157-167

Schendel SL, Xie Z, Montal MO, Matsuyama S, Montal M and Reed JC (1997) Channel Formation by Anti-Apoptotic Protein, Bcl-2. Proc. Natl. Acad. Sci. USA. 94: $5113-5118$

Shimizu S, EguchiY, Kamiike W, Matsuda Hand Tsujimoto Y (1996) Bcl-2 expression prevents activation of the ICE protease cascade. Oncogene 12: 2251-2257

Strasser A, Harris AW, Huang DC, Krammer PH and Cory S (1995) Bcl-2 and Fas/ APO-1 regulate distinct pathways to lymphocyte apoptosis. EMBO J. 14:61366147

Susin SA, Zamzami N, Castedo M, Hirsch T, Marchetti P, Macho A, Daugas E, Geuskens M and Kroemer G (1996) Bcl-2 inhibits the mitochondrial release of an apoptogenic protease. J. Exp. Med. 184: 1331-1341

Vayssiere JL, Petit PX, Risler Y and Mignotte B (1994) Commitment to apoptosis is associated with changes in mitochondrial biogenesis and activity in cell lines conditionally immortalized with simian virus 40 . Proc. Natl. Acad. Sci. USA. 91 : $11752-11756$

White K, Tahaoglu E and Steller H (1996) Cell killing by the Drosophila gene reaper. Science 271: 805-807

Xiang J, Chao DT and Korsmeyer SJ (1996) BAX-induced cell death may not require interleukin $1 \beta$-converting enzyme-like proteases. Proc. Natl. Acad. Sci. USA. 93: $14559-14563$

Yang J, Liu X, Bhalla K, Kim CN, Ibrado AM, Cai J, Peng TI, Jones DP and Wang X (1997) Prevention of apoptosis by Bcl-2: release of cytochrome $c$ from mitochondria blocked. Science 275: 1129-1132

Zamzami N, Marchetti P, Castedo M, Zanin C, Vayssiere JL, Petit PX and Kroemer G (1995) Reduction in mitochondrial potential constitutes an early irreversible step of programmed lymphocyte death in vivo. J. Exp. Med. 181: 1661-1672 